\title{
Threshold criterion for wetting at the triple point
}

\author{
S. Curtarolo ${ }^{1,3}$, G. $\operatorname{Stan}^{1}$, M. J. Bojan ${ }^{2}$, M. W. Cole ${ }^{1,4}$, and W. A. Steele ${ }^{2}$ \\ ${ }^{1}$ Department of Physics, Penn State University, University Park, PA 16802, USA \\ ${ }^{2}$ Department of Chemistry, Penn State University, University Park, PA 16802, USA \\ ${ }^{3}$ Present address: Dept. of Materials Science and Engineering, MIT, Cambridge, MA 02139, USA \\ ${ }^{4}$ corresponding author, e-mail: mwc@psu.edu, phone: (814) 863-0165, fax: (814) 865-3604
}

(May 11, 2018)

\begin{abstract}
Grand canonical simulations are used to calculate adsorption isotherms of various classical gases on alkali metal and $\mathrm{Mg}$ surfaces. Ab initio adsorption potentials and Lennard-Jones gas-gas interactions are used. Depending on the system, the resulting behavior can be nonwetting for all temperatures studied, complete wetting, or (in the intermediate case) exhibit a wetting transition. An unusual variety of wetting transitions at the triple point is found in the case of a specific adsorption potential of intermediate strength. The general threshold for wetting near the triple point is found to be close to that predicted with a heuristic model of Cheng et al. This same conclusion was drawn in a recent experimental and simulation study of $\mathrm{Ar}$ on $\mathrm{CO}_{2}$ by Mistura et al. These results imply that a dimensionless wetting parameter $w$ is useful for predicting whether wetting behavior is present at and above the triple temperature. The nonwetting/wetting crossover value found here is $w \simeq 3.3$.
\end{abstract}

PACS numbers: 64.70.Fx, 68.35.Rh, 68.45.Gd, 82.20.Wt 


\section{INTRODUCTION}

Many adsorption systems exhibit either complete wetting below the triple point or triple point wetting transitions. These behaviors are recognized to arise from strongly attracting substrate potentials and/or small mismatch of substrate/adsorbate lattice constants [1,2]. Less attractive potentials exhibit incomplete or nonwetting behavior at the triple point. There is considerable interest in the nature of such weakly attractive situations near and above this temperature [3]. General arguments and model calculations of Cahn [4] and of Ebner and Saam [5] in 1977 implied that systems which are nonwetting at the triple point ought to exhibit transitions to wetting at some temperature below the critical point. Experimental confirmation of this proposal was not found until appropriate systems were identified. In 1991, it was argued that alkali metals provide the weakest adsorption potentials of any surfaces for He atoms and therefore wetting transitions were expected for such substrates at tow temperature [6]. This hypothesis was subsequently confirmed and the corresponding transition has since been studied in many laboratories [7 9]. More recently, similar phenomena have been predicted and/or seen for $\mathrm{H}_{2}, \mathrm{Ne}$, and $\mathrm{Hg}$ on various surfaces 10 15. Key theoretical questions arising from these studies involve the reliability of both the assumed adsorption potentials and the statistical mechanical description of the transition, as well as the effects of surface heterogeneity [16] present in any real experiment.

In the course of these investigations, Cheng et al. [6] (CCST henceforth) posited a simple model which interprets the transitions in terms of a balance between the surface tension cost of producing a thicker film and the energy gain associated with the film's interaction with the surface, $\mathrm{V}(\mathrm{z})$ [17]. There results an implicit equation for the wetting temperature $\left(\mathrm{T}_{w}\right)$ :

$$
\begin{gathered}
\left(\rho_{l}-\rho_{v}\right) I_{V}=2 \gamma \\
I_{V}=-\int_{z_{\text {min }}}^{\infty} d z V(z)
\end{gathered}
$$

where $\rho_{l}$ and $\rho_{v}$ are the number densities of the adsorbate liquid and vapor at coexistence, 
$\gamma$ is the surface tension of the liquid, and $z_{\min }$ is the equilibrium distance of the potential. If one assumes a simple 3-9 form of the adsorption potential

$$
V=\left(4 C^{3}\right) /\left(27 D^{2} z^{9}\right)-C / z^{3}
$$

then the transition condition becomes

$$
\left(C D^{2}\right)^{1 / 3}=3.33 \gamma /\left(\rho_{l}-\rho_{v}\right)
$$

CCST used this model to predict wetting transitions for a large number of specific systems. More recently, the same general approach was used by Chizmeshya et al. (CCZ) 18], who based their predictions on new ab initio adsorption potentials. The latter potentials differ from earlier potentials in several respects; especially important are differences in values of the coefficient $\mathrm{C}$ of the van der Waals asymptotic potential, resulting in significantly larger well depths D. There is indication that these revised potentials are more consistent with experimental data than are their predecessors. Particularly striking is the improved agreement for ${ }^{4} \mathrm{He}$ contact angle [19 21] and wetting temperature on Cs, the most studied wetting transition to date [7] 9].

These predictions have also been tested by recent experiments of Hess et al. [13 and simulations of $\mathrm{Ne}$ on alkali metals and $\mathrm{Mg}$ [22]. In this paper, we extend the simulation studies to a larger family of systems for which the hypothesis is relevant. We focus here on the following question: assuming that $\mathrm{V}(\mathrm{z})$ is known, does the CCST model correctly predict whether a specific adsorption system exhibits wetting at and above the triple temperature?

The outline of this paper is the following. Section [I] summarizes our simulation methodology and presents detailed results for several systems which yield fairly distinct adsorption behaviors. These include complete wetting for all $\mathrm{T}(\mathrm{Kr} / \mathrm{Mg}$ and $\mathrm{Xe} / \mathrm{Mg})$, an anomalous wetting transition near the triple point $(\mathrm{Kr} / \mathrm{PMg})$, a prewetting transition line $(\mathrm{Ar} / \mathrm{Mg})$ somewhat above the triple temperature, and prewetting transitions characterized by the formation of very thick films (for $\mathrm{Ar}, \mathrm{Kr}$ and $\mathrm{Xe}$ on $\mathrm{Li}$ ).

Here PMg is our own nomenclature for "pseudo-magnesium", an ersatz surface which attracts $\mathrm{Kr}$ atoms about $7 \%$ more strongly than $\mathrm{Mg}$ itself [23]. We also evaluate other 
hypothetical substrates in order to ascertain the triple point wetting threshold. In section III, we compare these findings with those obtained previously in simulations and/or experiments for inert gases on various surfaces [24 27.

\section{METHOD AND RESULTS}

The simulation technique has been described in detail previously [22], so only a brief description is given here. For this study, isotherms for various model systems were calculated using the Grand Canonical Monte Carlo (GCMC) technique [28,29]. For each isotherm, 6.8 million steps (each step being an attempted creation, deletion, or displacement of a molecule) were performed to reach equilibration and 4.5 million steps were performed in the data gathering phase. For some points near the transition regions, where the fluctuations are larger, the number of equilibration steps was increased to 20 million and 12 million steps were performed for data gathering.

As in our previous work, the model for the atom-atom interaction is a Lennard-Jones 12-6 potential and the atom surface interaction is modeled using the potential described by Chizmeshya et al. [18]. These potentials can be characterized by four parameters for each system studied. A well-depth $\epsilon_{g g}$ and distance $\sigma_{g g}$ are needed to specify the adsorbate LJ potential and a well-depth $D$ and the van der Waals constant $C$ specify the atom-surface potential. The values of these parameters used in this study are listed in Table $\mathbb{1}$. The parameters correspond to $\mathrm{Ne}, \mathrm{Kr}$, Ar, and Xe adsorbed on alkali and alkali earth metal surfaces. The surface labeled PMg (pseudo magnesium) was obtained when we attempted to simulate Xe on Li, but used the $\epsilon_{g g}$ and $\sigma_{g g}$ values for Kr instead of Xe. This potential is similar to that of $\mathrm{Kr}$ on a Mg surface; however, the $\mathrm{Kr} / \mathrm{PMg}$ distance of closest approach is $20 \%$ smaller and the well depth is about $7 \%$ weaker than estimated for $\mathrm{Kr}$ on Mg. While this potential does not correspond to a "real" system, we shall see that this small decrease in interaction strength significantly affects the wetting properties of the substrate/adsorbate system. 
In most cases the simulation cell had a height of $h=70 \AA$, with the metal surface forming the boundary at one end and a hard wall surface at the other. In Xe/Li and $\mathrm{Ar} / \mathrm{Li}$ simulations we increased the height to $140 \AA$ and $210 \AA$. The surface unit cell was taken to be $10 \sigma_{g g} \times 10 \sigma_{g g}$ giving a nominal monolayer coverage of roughly 100 atoms. Periodic boundary conditions in these dimensions render the surface effectively infinite; we used a fairly large cutoff $\left(5 \sigma_{g g}\right)$ to minimize long range corrections.

For each system, we simulated adsorption isotherms over relevant temperature ranges from the triple point to the critical temperature. The results are shown in figures 1 10 . Of the systems studied the least attractive surface of the surface interactions are for the $\mathrm{Ar} / \mathrm{Li}$, $\mathrm{Kr} / \mathrm{Li}$ and $\mathrm{Xe} / \mathrm{Li}$ cases. In all of these, we see evidence of prewetting transitions and, based on our results, we report wetting temperature. For $\mathrm{Ar} / \mathrm{Li}$ we estimate $\mathrm{T}_{w}=130 \pm 4 \mathrm{~K}$, for $\mathrm{Kr} / \mathrm{Li}, \mathrm{T}_{w}=175 \pm 4 \mathrm{~K}$ and $\mathrm{T}_{w}=225 \pm 4 \mathrm{~K}$ for Xe/Li. All $T_{w}$ are reported in Table $\mathbb{\llbracket}$.

Despite the fact that these systems are examples of prewetting transitions, there are some qualitative differences from the case (say $\mathrm{Ne} / \mathrm{Mg}$ ) where the wetting transition appears close to the triple temperature. Figure 1 shows a series of isotherms for $\mathrm{Xe} / \mathrm{Li}$ from $T^{*}=1.0$ to 1.25. The isotherm at $221 \mathrm{~K}$ (the lowest $T^{*}$ ) shows nonwetting behavior, manifested as slight adsorption below saturated vapor pressure. The higher temperature isotherms however, show prewetting transitions below saturated vapor pressure, from which we estimate $T_{w}$. The nature of the prewetting jump can be further characterized by the results in figures 2. 23, and 田, all at $\mathrm{T}=254 \mathrm{~K}$. Figure $\mathrm{Q}$ shows the dependence of the isotherm on the height $h[=70,140,210 \AA]$ of the simulation box. The rapid rise occurs at the same pressure, independent of box height. This is a clear indication that the transition is prewetting rather than capillary condensation. The detailed behavior is most easily understood by looking at the density profiles' dependences on $h$. Figure [3 shows these profiles at $P=22.9 \mathrm{~atm}$. Note that the data for two largest boxes coincide but differ from those of the smallest box. Evidently, this regime requires quite large values of $h$ in order to obtain reliable data; this is not surprising because the compressibility of the system is particularly high at this transition 
as the slope of the isotherms suggest. The same phenomenon is even more dramatic for the case of a $1 \%$ higher pressure. As seen in figure $\mathbb{t}$, one must go to even larger $h(>200$ $\AA$ ) in order to derive accurate adsorption data even though the liquid regime occupies only the region $z<70 \AA$. While this sensitivity to $h$ and the details of the transition are quite interesting they are not the focus of this paper. Suffice it to say that the density jumps discontinuously near $P=23.0 \mathrm{~atm}$, as seen in the different densities in figures $\mathrm{B}$ and 1 .

We note one further point about these data which is that the film's density close to the surface is qualitatively similar to that on a relatively more attractive surface. There appears a quite large accumulation of adsorbate atoms in the region associated with the first layer. There is even a significant density maximum occurring where a dense second layer might occur. The latter is, however, preempted by the prewetting jump in the regime of temperature seen here. Above the prewetting critical point, in contrast, one does expect such smooth film growth.

As the relative strength $\left(D / \epsilon_{g g}\right)$ of the surface interaction increases, ( $\mathrm{Ar}$ on $\mathrm{Mg}, \mathrm{Kr}$ on $\mathrm{PMg}$ ) the isotherms give evidence of wetting transitions. The results for $\mathrm{Ar}$ on $\mathrm{Mg}$, (shown in figure 5) indicate prewetting transitions at about $10 \%$ above the triple point $\left(\mathrm{T}_{t r}=81\right.$ $\mathrm{K})$. From these data, we estimate the wetting temperature to be $90 \mathrm{~K}$ for $\mathrm{Ar} / \mathrm{Mg}$ with a prewetting critical temperature of $95 \mathrm{~K}$.

In figure 6, the results for $\mathrm{Kr}$ on $\mathrm{PMg}$ are shown. This system shows clear prewetting transitions below $128 \mathrm{~K}$ for all temperatures above the triple temperature (estimated to be $116 \mathrm{~K}$ [34]). Although this is a fictitious system, it represents one of the first simulations of triple point wetting. The only other simulation of wetting in this region is the case of $\mathrm{Ne} / \mathrm{Mg}$ which we reported in 22.

Finally, as the surface interaction strength further increases, one begins to see continuous growth for all $\mathrm{T}$ between the triple temperature and the critical point. This is evident in figure 7 which shows isotherms for $\mathrm{Kr}$ on $\mathrm{Mg}$. The isotherms were simulated at temperatures near the estimated triple temperature and demonstrate complete wetting on the Mg surface by the adsorbate even at the lowest temperatures. The results for Xe on $\mathrm{Mg}$ (not shown) 
are qualitatively the same.

\section{INTERPRETATION OF THE RESULTS}

To interpret our results we follow CCST, who evaluated their simple model in terms of the law of corresponding states. Then we note that equation 1 may be written in a universal form in terms of the reduced well depth $D^{*}$ and interaction range $x$, defined as follows:

$$
\begin{aligned}
D^{*} & =D / \epsilon_{g g} \\
x & =(C / D)^{1 / 3} / \sigma_{g g}
\end{aligned}
$$

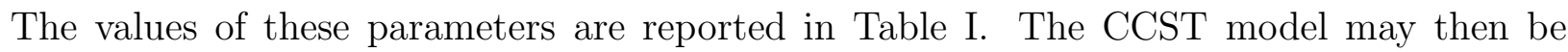
written in terms of a dimensionless wetting parameter

$$
w=x D^{*}=3.33 \gamma /\left[\left(\rho_{l}-\rho_{v}\right) \sigma_{g g} \epsilon_{g g}\right]
$$

The triple point experimental data summarized in CCST yield a value of 3.6 for the right side of this equation. Hence the criterion is $w=3.6$ for a wetting transition precisely at the triple point. The latter value, however, presupposes a 3-9 adsorption potential, which we have not used in the present simulations. If, instead, we compute the integral of the actual potential used in the calculations, we find a value which is 8 to $10 \%$ higher than the result obtained with the 3-9 potential. Taking this correction into account, the threshold for wetting at the triple point becomes somewhat lower for the realistic potential:

$$
w=3.3
$$

This is shown in figure 8 as a dashed curve. Turning to the actual simulation results, we find that this prediction characterizes our data relatively well, insofar as the vicinity of the value 3.3 is a crossover regime of wetting behavior. For all gases exposed to the alkali metal surfaces the quantity $w$ is less than 2.3, and the systems are nonwetting at the triple point. The case of $\operatorname{Ar} / \mathrm{Mg}(w=3.2)$ is one for which a wetting transition occurs about $10 \%$ above 
the triple temperature; similar behavior was found in our previous study of $\mathrm{Ne} / \mathrm{Mg}$ ( $w=$ 3.4). The case of $\mathrm{Kr} / \mathrm{PMg}$ involves a similar value $(w=3.2)$, but we find anomalous triple point wetting transition behavior for that system. Finally, the cases of $\mathrm{Kr} / \mathrm{Mg}(w=3.4)$ and $\mathrm{Xe} / \mathrm{Mg}(w=3.8)$ both correspond to complete wetting. For comparison with a more typical adsorption system, the case of $\operatorname{Ar} / \mathrm{Au}(w=6.7)$ is one which exhibits wetting at and above the triple temperature 24,27].

We may add to this list of relevant systems the recently studied case of $\mathrm{Ar} / \mathrm{CO}_{2}$. Mistura et al. [25] found that it exhibits triple point wetting; this finding agreed with their density functional calculation using their theoretical potential. This potential would be roughly consistent with the CCST model in that the latter would predict a wetting transition at a temperature only $3 \%$ above the triple point temperature, $83.8 \mathrm{~K}$ [30].

While the criterion $w=3.3$ successfully discriminates between the nonwetting and wetting data sets mentioned above, it is worthwhile to ascertain the validity of this criterion more accurately. Hence we have performed extensive simulations at the triple temperature, varying the well depth near the values $x=0.7,0.9$, and 1.4 in order to determine the wetting transition line. The results are shown in Figure 8 . One identifies this line as the point where the letter $\mathrm{C}$ is just above the letter W. Note that these transition values (dash-dot curve) lie somewhat above the $w=3.3$ dashed line. This comparison indicates that the CCST model underestimates the well depth criterion for wetting.

This finding is qualitatively consistent with results reported recently by Ancilotto and Toigo [35]. Using a density functional method, these workers found wetting transitions very close to the coexistence curve for several systems (e.g. Ne/Li and Na) for which our simulations find nonwetting behavior. The different findings of the simulations may be due to the uncertainty in identifying wetting behavior close to saturation, especially at $\mathrm{T}$ approaching $\mathrm{T}_{c}$. In these and other cases, the transitions occurred at (typically $\sim$ $10 \%$ ) higher temperature than is predicted by the CCST model. These findings are also consistent with one's realization that the CCST model predicts wetting transitions for all adsorption circumstances, however weakly attractive, while a more reliable theory yields 
drying transitions for ultraweakly adsorbing potentials [35].

To summarize, we have found the CCST equation 2 to be a semiquantitative guide to the occurrence of wetting transitions. It would be valuable to have additional, genuine experimental data to test this conclusion. We wish especially to encourage experimentalists to explore totally new systems, especially Ar, Kr, and Xe adsorption on alkali metals. There are several reasons for doing so. One is that such experiments will provide tests of the weak adsorption potentials for these systems, for which no experimental data exist. A second is that triple point wetting can involve interesting behavior at and just below the triple temperature, as has been found in many experiments 31,36.

We have not explored either these systems, or analogous ones, below the triple tem-

perature. In order to reliably describe the crystalline phase, as is necessary there, such simulations would require a more careful handling of the unit cell dimensions and shape than has been employed here. In view of the many relevant systems, such a study seems well justified for the future.

\section{ACKNOWLEDGMENTS}

This research was supported by the National Science Foundation. It has benefited from discussion with F. Ancilotto, V. Bakaev, M. H. W. Chan, J. Z. Larese, G. Mistura, and F. Toigo. We are especially grateful to Fondazione Aldo Ing. Gini for the generous support of Ing. S. Curtarolo. 


\section{TABLES}

TABLE I. The values of the LJ parameters, $\epsilon_{g g}$ and $\sigma_{g g}$, for the gas-gas interactions and the values of the reduced well depth and width $D^{*}=D / \epsilon_{g g}$ and $x$, as defined in Eq. (6), for various systems studied and correlated with wetting behavior, defined as follows: $\mathrm{C}=$ continuous growth, $\mathrm{TP}=$ anomalous wetting transition near or at the triple point $\left(\mathrm{T}_{t r}\right), \mathrm{W}=$ wetting transition above the triple point, $\mathrm{N}=$ nonwetting for all $\mathrm{T}>\mathrm{T}_{t r}$, and $\mathrm{D}=$ drying. The $\mathrm{Ar} / \mathrm{CO}_{2}$ data were taken from Ref. [27]. The wetting temperatures estimated by the simulations are in parentheses.

\begin{tabular}{|c|c|c|c|c|c|c|c|}
\hline Gas / Surface & & $\mathrm{Rb}$ & $\mathrm{Na}$ & $\mathrm{Li}$ & $\mathrm{PMg}$ & $\mathrm{Mg}$ & $\mathrm{CO}_{2}$ \\
\hline $\mathrm{Ne}$ & $D^{*}$ & 0.7 & 1.1 & 1.5 & & 2.8 & \\
\hline$\epsilon_{g g}=34 \mathrm{~K}$ & $x$ & 1.6 & 1.4 & 1.4 & & 1.2 & \\
\hline \multirow[t]{2}{*}{$\sigma_{g g}=2.78 \AA$} & behavior & $\mathrm{D}$ & $\mathrm{N}$ & $\mathrm{N}$ & & $\mathrm{TP}(22 \mathrm{~K})$ & \\
\hline & $x D^{*}$ & 1.1 & 1.5 & 2.1 & & 3.4 & \\
\hline $\mathrm{Ar}$ & $D^{*}$ & 1.1 & 1.6 & 2.1 & & 3.5 & 3.8 \\
\hline$\epsilon_{g g}=120 \mathrm{~K}$ & $x$ & 1.1 & 1.1 & 1.0 & & 0.9 & 1.0 \\
\hline \multirow[t]{2}{*}{$\sigma_{g g}=3.41 \AA$} & behavior & $\mathrm{N}$ & $\mathrm{N}$ & $\mathrm{W}(130 \mathrm{~K})$ & & $\mathrm{W}(90 \mathrm{~K})$ & $\mathrm{C}$ \\
\hline & $x D^{*}$ & 1.2 & 1.7 & 2.1 & & 3.2 & 3.8 \\
\hline $\mathrm{Kr}$ & $D^{*}$ & & & 2.3 & 3.6 & 3.8 & \\
\hline$\epsilon_{g g}=171 \mathrm{~K}$ & $x$ & & & 0.9 & 0.9 & 0.9 & \\
\hline \multirow[t]{2}{*}{$\sigma_{g g}=3.60 \AA$} & behavior & & & $\mathrm{W}(175 \mathrm{~K})$ & $\mathrm{TP}(116 \mathrm{~K})$ & $\mathrm{C}$ & \\
\hline & $x D^{*}$ & & & 2.1 & 3.2 & 3.4 & \\
\hline $\mathrm{Xe}$ & $D^{*}$ & & & 2.8 & & 5.45 & \\
\hline$\epsilon_{g g}=221 \mathrm{~K}$ & $x$ & & & 0.8 & & 0.7 & \\
\hline \multirow[t]{2}{*}{$\sigma_{g g}=4.10 \AA$} & behavior & & & $\mathrm{W}(225 \mathrm{~K})$ & & $\mathrm{C}$ & \\
\hline & $x D^{*}$ & & & 2.2 & & 3.8 & \\
\hline
\end{tabular}




\section{REFERENCES}

[1] R. Pandit, M. Schick, and M. Wortis, Phys. Rev. B 26, 5112, 1982; S. Dietrich in Phase Transitions and Critical Phenomena, C. Domb and J. L. Lebowitz, Eds., (Academic, London) 12, 1 (1998).

[2] P. G. de Gennes, Rev. Mod. Phys. 57, 827 (1985).

[3] M. W. Cole, Fluid Phase Equilibria 150, 559 (1998).

[4] J. W. Cahn, J. Chem. Phys. 66, 3667 (1977).

[5] C. E. Ebner and W. F. Saam, Phys. Rev. Lett. 38, 1486 (1977).

[6] E. Cheng, M. W. Cole and W. F. Saam and J. Treiner, Phys. Rev. Lett. 67, 1007 (1991); Phys. Rev. B 46, 13967 (1992); Erratum B 47, 14661 (1993).

[7] P.-J. Nacher and J. Dupont-Roc, Phys. Rev. Lett. 67, 2966 (1991); P. J. Nacher, B. Demolder, and J. Dupont-Roc, Physica B 194, 975 (1994).

[8] J. Rutledge and P. Taborek, Phys. Rev. Lett. 69, 937 (1992); J. E. Rutledge and P. Taborek, J. Low Temp. Phys. 95, 405 (1994); D. Ross, J. A. Phillips, and P. Taborek, J. Low Temp. Phys. 106, 81 (1997); D. Reinelt, H. Gau, S. Herminghaus and P. Leiderer, Czech. J. Phys. 46, Suppl. S1, 431 (1996).

[9] K. S. Ketola, S. Wang, and R. B. Hallock, Phys. Rev. Lett. 68, 201 (1992); R. B. Hallock, J. Low Temp. Phys. 101, 31 (1995); A. F. G. Wyatt, J. Klier, and P. Stefanyi, Phys. Rev. Lett. 74, 1151 (1995).

[10] E. Cheng, G. Mistura, H. C. Lee, M. H. W. Chan, M. W. Cole, C. Carraro, W. F. Saam and F. Toigo, Phys. Rev. Lett. 70, 1854 (1993).

[11] D. Ross, J. E. Rutledge, and P. Taborek, Fluid Phase Equilibria 150-151, 599 (1998); D. Ross, J. E. Rutledge, and P. Taborek, Science 278, 664 (1997); J. A. Phillips, P. Taborek, and J. E. Rutledge, J. Low Temp. Phys. 113, 829 (1998); J. A. Phillips, 
D. Ross, P. Taborek, and J. E. Rutledge, Phys. Rev. B 58, 3361 (1998); D. Ross, P. Taborek, and J. E. Rutledge, Phys. Rev. B 58, R4274 (1998).

[12] G. Mistura, H. C. Lee and M. H. W. Chan, J. Low Temp. Phys. 96, 221 (1994).

[13] G. B. Hess, M. J. Sabatini and M. H. W. Chan, Phys. Rev. Lett. 78, 1739 (1997).

[14] F. Hensel and M. Yao, Eur. J. Sol. Stat. Inorg. Chem. 34, 861 (1997).

[15] V. F. Kozhevnikov, D. I. Arnold, S. P. Naurzakov, and M. E. Fisher, Fluid Phase Equilibria 150-151, 625 (1998).

[16] S. Curtarolo, G. Stan, M. W. Cole, M. J. Bojan, and W. A. Steele, Phys. Rev. E 59, $4402(1999)$.

[17] E. Cheng, M. W. Cole, W. F. Saam and J. Treiner, Phys. Rev. B 48, 18214 (1993).

[18] A. Chizmeshya, M. W. Cole, and E. Zaremba, J. Low Temp. Phys. 110, 677 (1998).

[19] D. Ross, P. Taborek, and J. E. Rutledge, J. Low Temp. Phys. 111, 1 (1998).

[20] J. Klier and A. F. G. Wyatt, J. Low Temp. Phys. 110, 919 (1998).

[21] F. Ancilotto, A. M. Sartori, and F. Toigo, Phys. Rev. B 58, 5085 (1998).

[22] M. J. Bojan, G. Stan, S. Curtarolo, W. A. Steele, M. W. Cole, Phys. Rev. E 59, 864 (1999).

[23] The alert reader might notice that the adsorption potential parameters for $\mathrm{Kr} / \mathrm{PMg}$ coincide with those for $\mathrm{Xe} / \mathrm{Li}$; this reveals the accidental origin of this serendipitous choice of fictitious substrate.

[24] K. G. Sukhatme, J. E. Rutledge, and P. Taborek, Phys. Rev. Lett. 80, 129 (1998).

[25] G. Mistura, F. Ancilotto, L. Bruschi, and F. Toigo, Phys. Rev. Lett. 82, 795 (1999).

[26] L. Bruschi and G. Mistura, Phys. Rev. B 58, 1181 (1998). 
[27] J. Krim, J. G. Dash, and J. Suzanne, Phys. Rev. Lett. 52, 640 (1984).

[28] D. Frenkel and B. Smit, Understanding Molecular Simulation. (From Algorithms to Applications), Academic Press, San Diego, 1996.

[29] M. P. Allen and D. J. Tildesley, Computer Simulation of Liquids, Oxford Univ. Press, Oxford, 1987.

[30] Prior to the recent work of Mistura et al., much theoretical attention had been devoted to the $\mathrm{Ar} / \mathrm{CO}_{2}$ problem, but the assumed potential was less attractive than that implied by the recent study. See Refs. [5, 25, 26].

[31] G. Zimmerli and M. H. W. Chan, Phys. Rev. B 45, 9347 (1992).

[32] J. E. Finn and P. A. Monson, Phys. Rev. A 39, 6402 (1989).

[33] E. Bruno, C. Caccamo, and P. Tarazona, Phys. Rev. A 35, 1210 (1987).

[34] This estimate is based on the results of J. P. Hansen and L. Verlet, Phys. Rev. 184, 151 (1969), who found the reduced triple point temperature for a Lennard-Jones fluid to be $0.68+/-0.02$.

[35] F. Ancilotto and F. Toigo, "Prewetting transitions of Ar and Ne on alkali metal surfaces", submitted to Phys. Rev.

[36] A. D. Migone, J. G. Dash, M. Schick, and O. E. Vilches, Phys. Rev. B 34, 6322 (1986); A. D. Migone, A. Hoffmann, J. G. Dash, and O. E. Vilches, Phys. Rev. B 37, 5440 (1988); M. S. Pettersen, M. J. Lysek and D. L. Goodstein, Surf. Sci. 175, 141 (1986); L. Bruschi and G. Mistura, Phys. Rev. B 58, 1181 (1998); J. Z. Larese and M. Y. M Lee, Phys. Rev. Lett. 79, 689 (1997). 


\section{FIGURES}

FIG. 1. Solid curves are adsorption isotherms for Xe/Li at T=221, 232, 243, 254, 265, $276 \mathrm{~K}$. The vertical dashed lines to the right of each curve indicate the saturated vapor pressures.

FIG. 2. Adsorption isotherms for $\mathrm{Xe} / \mathrm{Li}$ at $\mathrm{T}=254 \mathrm{~K}$ as a function of simulation cell height.

The heights for the curves shown are $70 \AA$ (dashed), $140 \AA$ (dots), $210 \AA$ (full curve). The vertical dashed line to the right indicates the saturated vapor pressure.

FIG. 3. Film density for $\mathrm{Xe} / \mathrm{Li}$ at $\mathrm{T}=254 \mathrm{~K}$ and $\mathrm{P}=22.9 \mathrm{~atm}$ as a function of reduced distance. Curves correspond to varying heights of the simulation cell: $h=70 \AA$ (dots), $140 \AA$ (dashed), 210 $\AA$ (dot-dashed), and $280 \AA$ (full curve). Note the latter three results coincide.

FIG. 4. Film density for $\mathrm{Xe} / \mathrm{Li}$ at $\mathrm{T}=254 \mathrm{~K}$ and $\mathrm{P}=23.1 \mathrm{~atm}$ as a function of reduced distance. Curves correspond to varying heights of the simulation cell: $h=70 \AA$ (dots), $140 \AA$ (dashed), 210 $\AA$ (dot-dashed), and $280 \AA$ (full curve). Note the latter two results coincide.

FIG. 5. Adsorption of $\mathrm{Ar}$ on $\mathrm{Mg}$ for $\mathrm{T}=89,90,91,92,94,95,96$, and $98 \mathrm{~K}$ (from left to right). The data indicate a wetting transition near $90 \mathrm{~K}$ and a prewetting critical point near 95 K. The vertical dashed lines occur at saturated pressure for each temperature.

FIG. 6. Adsorption of $\mathrm{Kr}$ on "pseudo-Mg", an artificial problem described in the text. The isotherms (from left) begin at $119 \mathrm{~K}$ and run to $128 \mathrm{~K}$. There is a wetting transition close to the triple temperature, $\mathrm{T}_{t r}=116 \mathrm{~K}$. The prewetting critical temperature is $127 \mathrm{~K}$.

FIG. 7. Adsorption of $\mathrm{Kr}$ on $\mathrm{Mg}$, showing continuous growth of the film for all $\mathrm{T}$ above the triple temperature. Curves correspond to $\mathrm{T}=120,125,130,135,140$, and $145 \mathrm{~K}$, from left to right. 
FIG. 8. Points represent systems shown in Table 1 or artificial systems studied as discussed in Section III. Symbols are defined in the caption to this table. The threshold line based on the model discussed in the text, $w=D^{*} x=3.3$, is indicated by dashes. The dash-dot curve represents the wetting threshold derived from our simulations. 


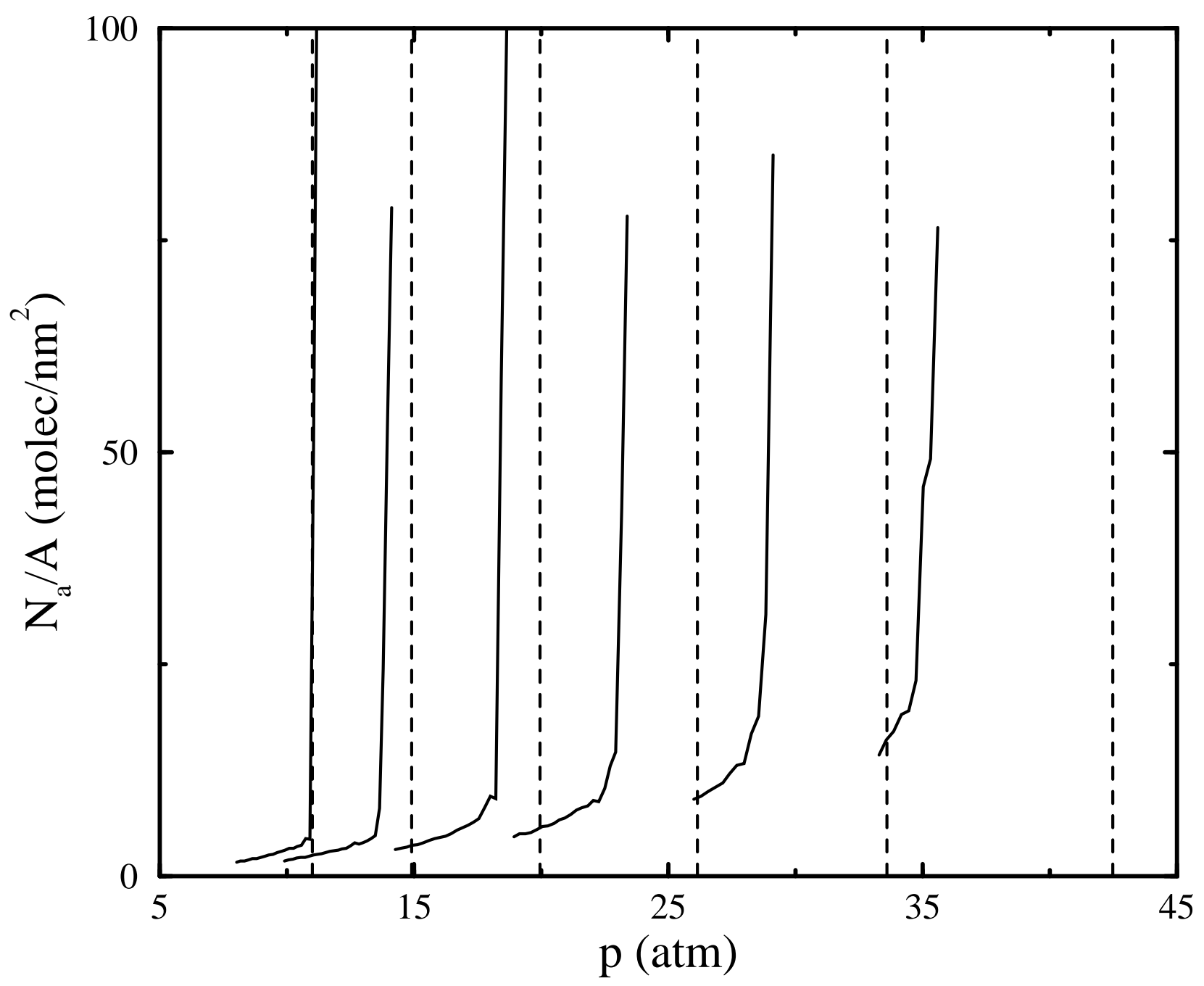

FIG. 1 


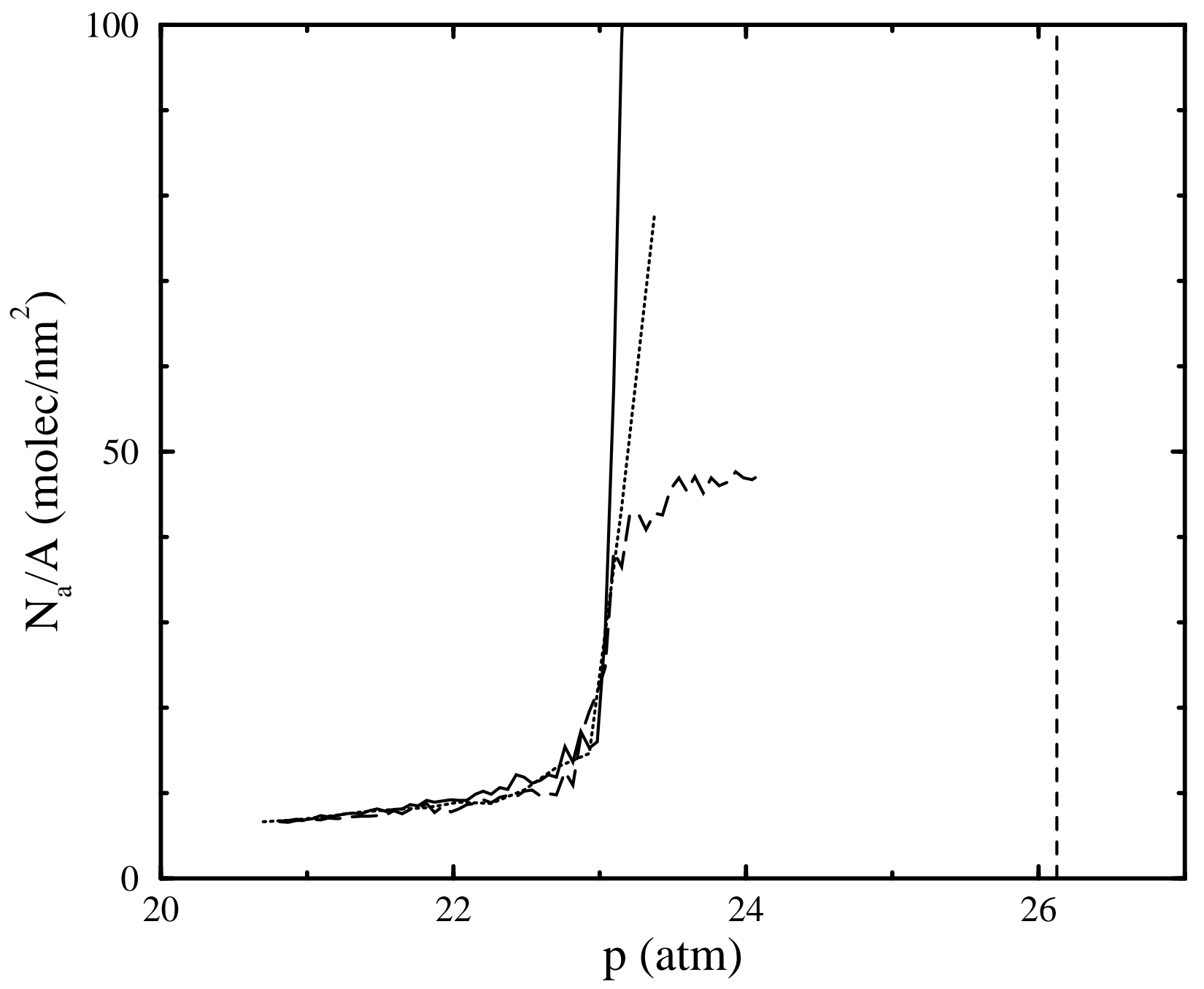

FIG. 2 


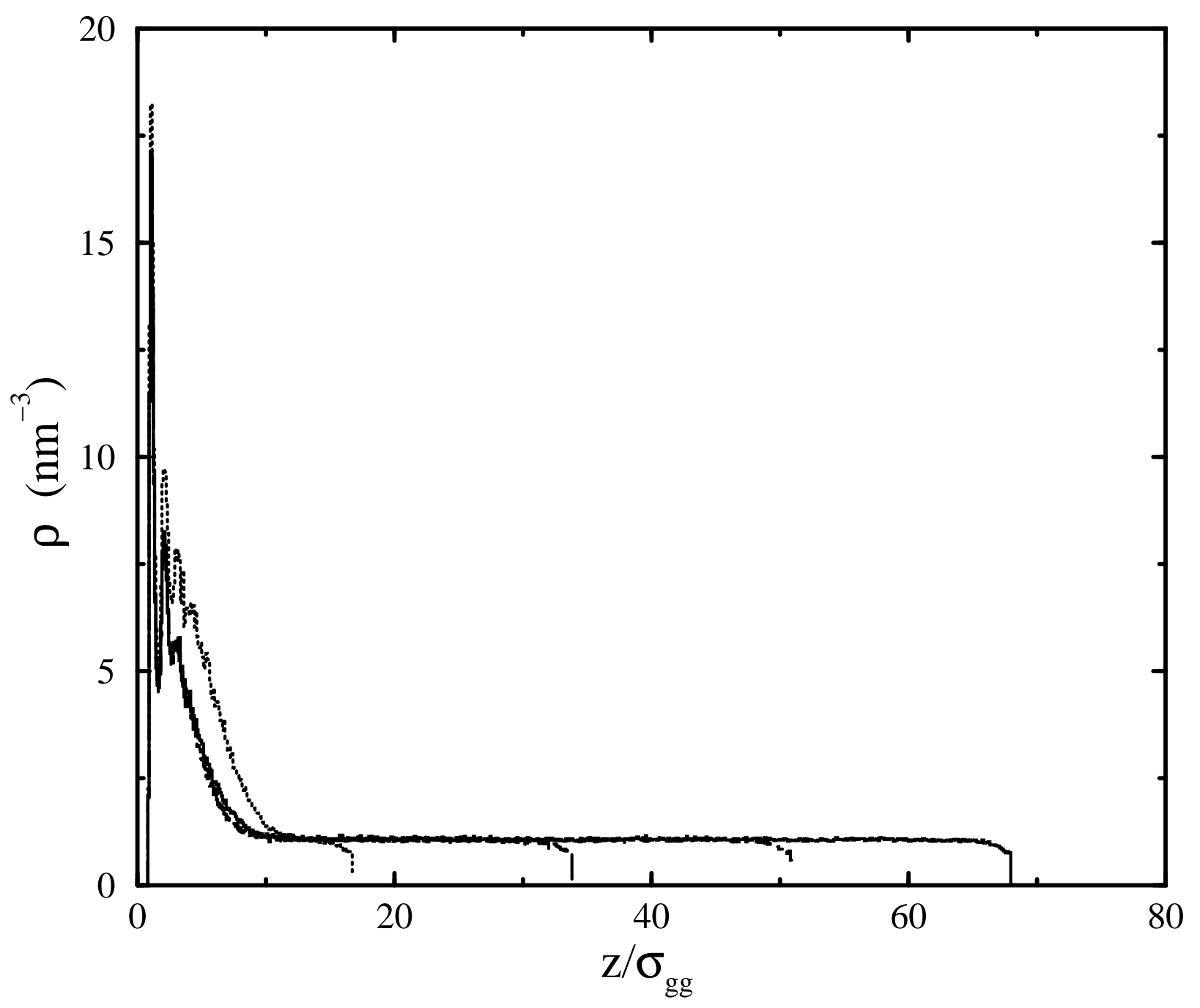

FIG. 3 


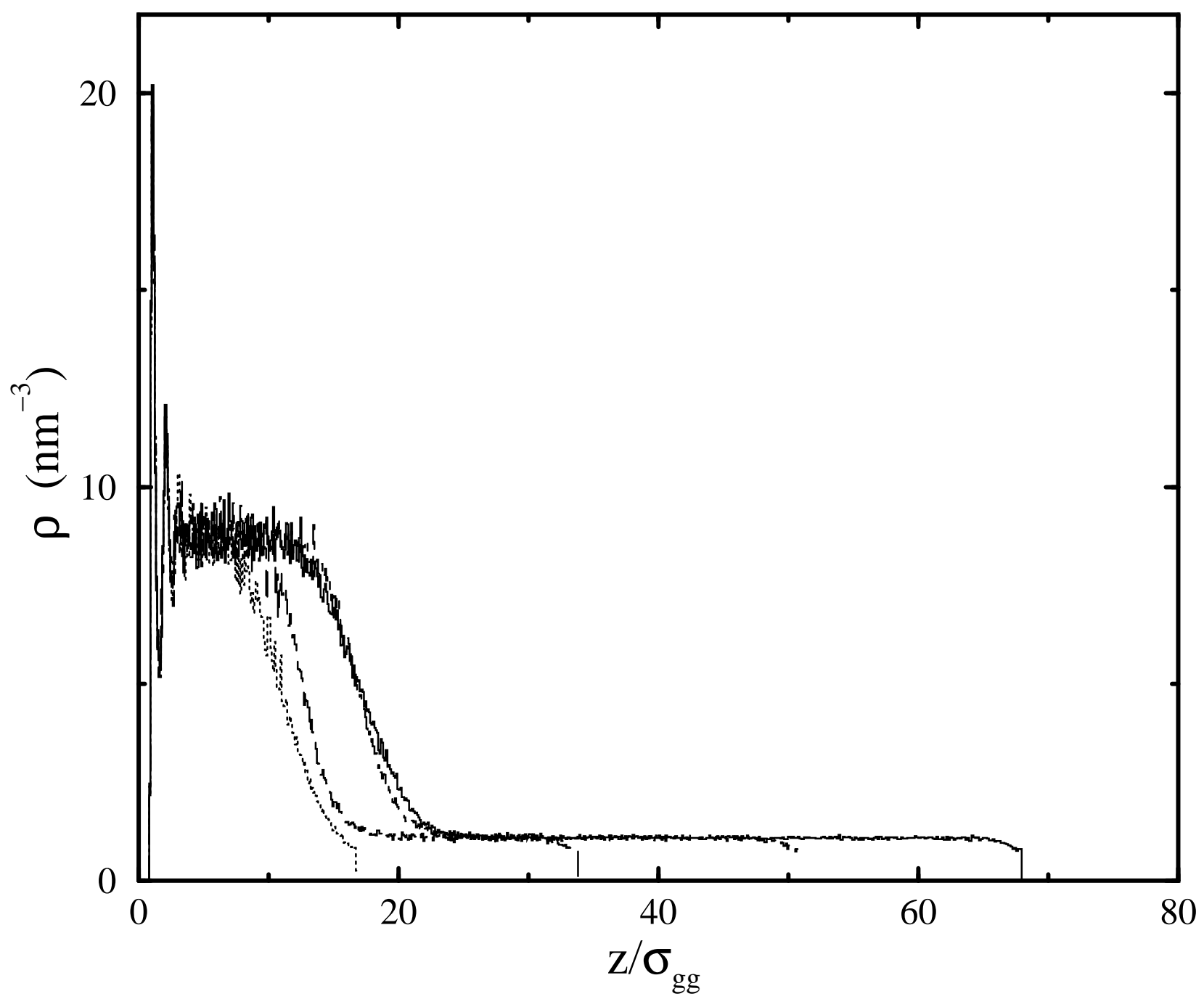

FIG. 4 


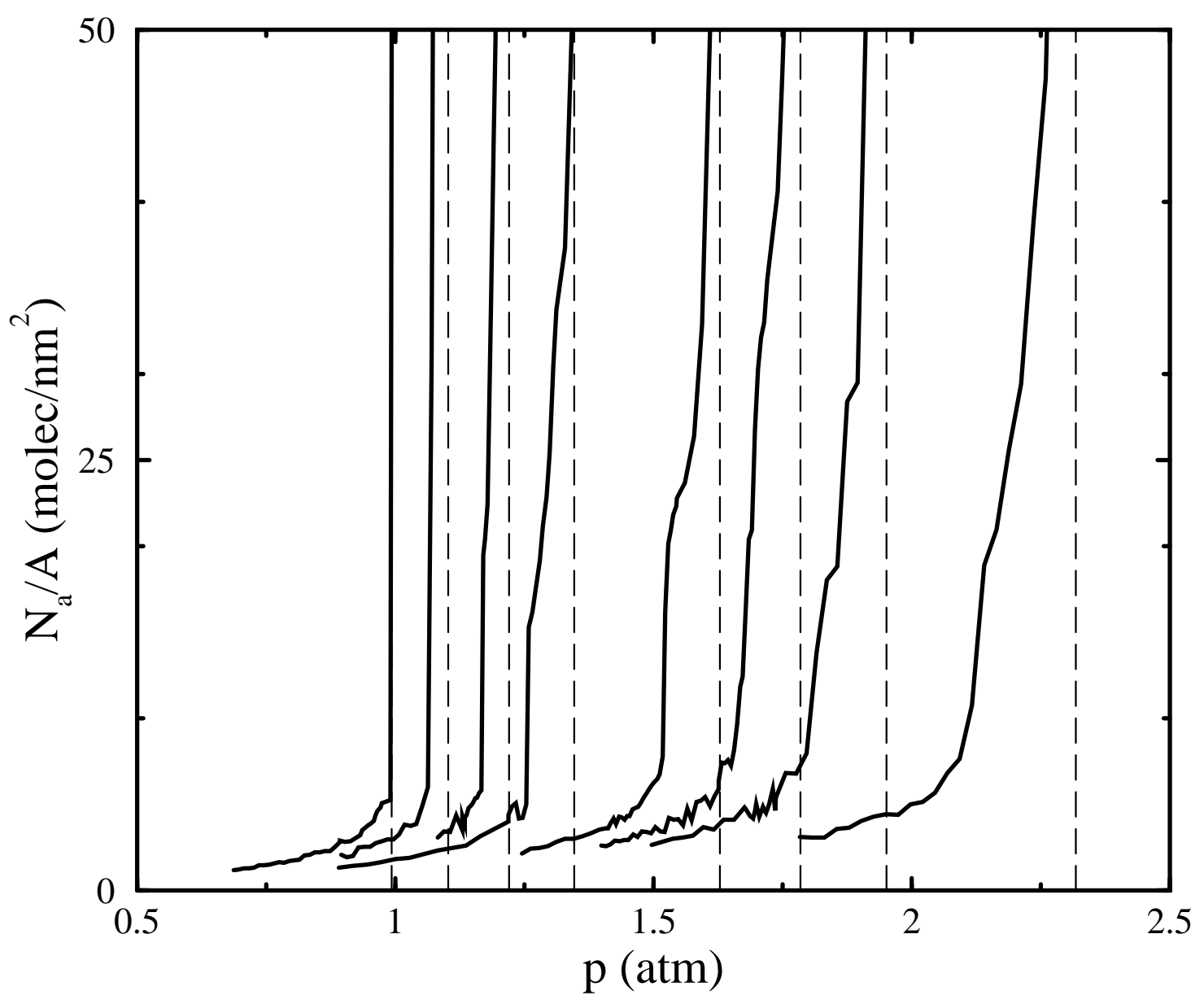

FIG. 5 


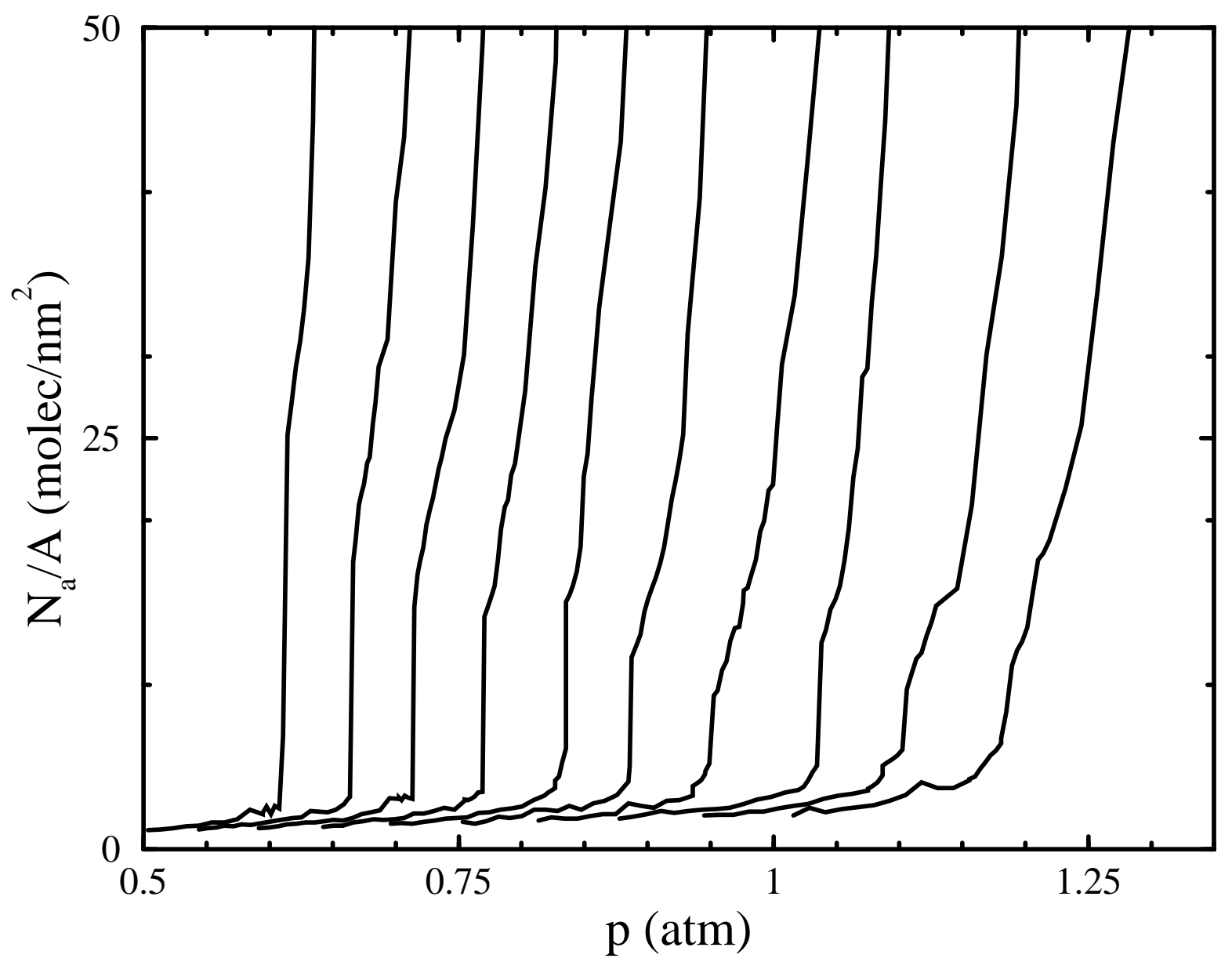

FIG. 6 


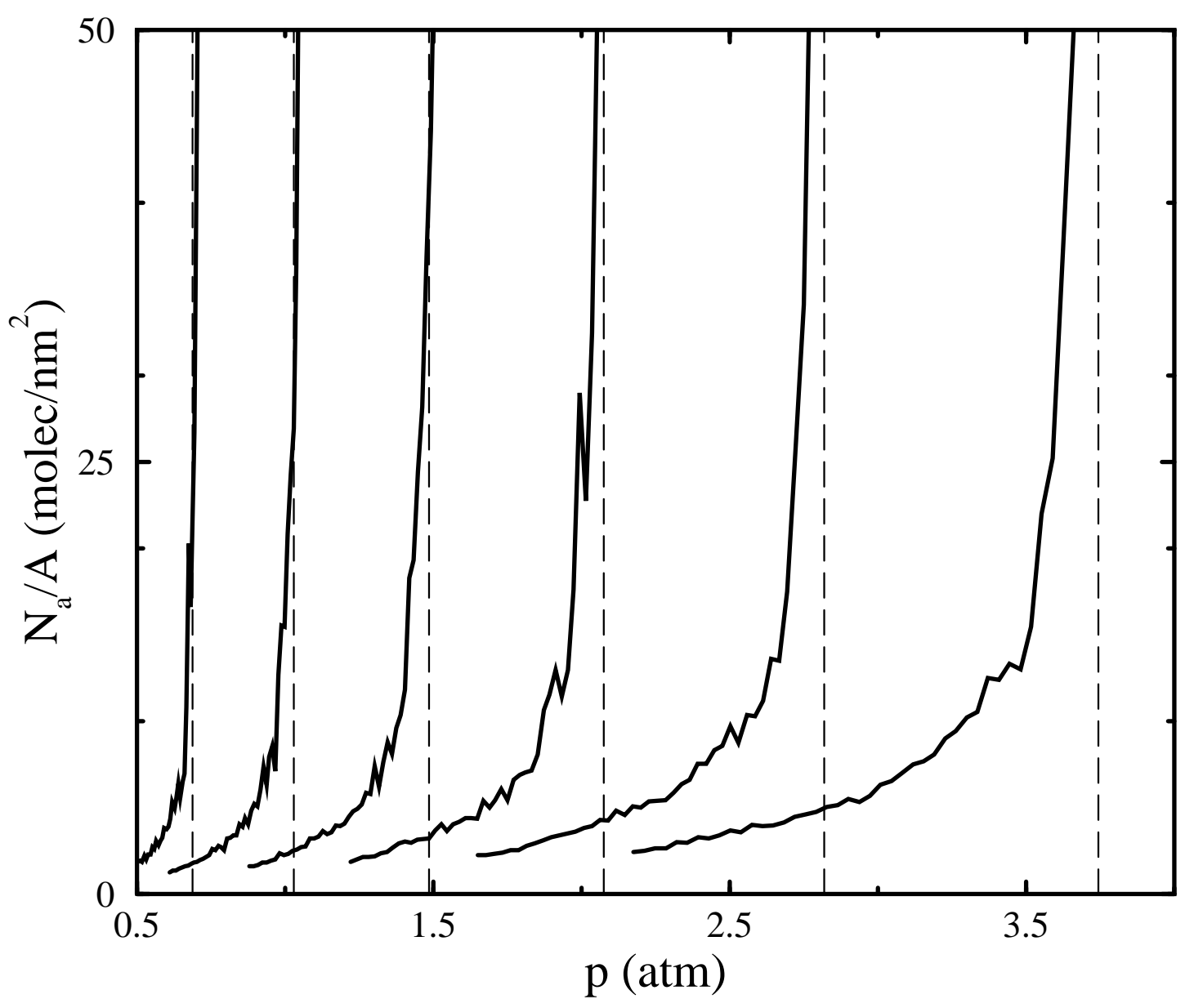

FIG. 7 


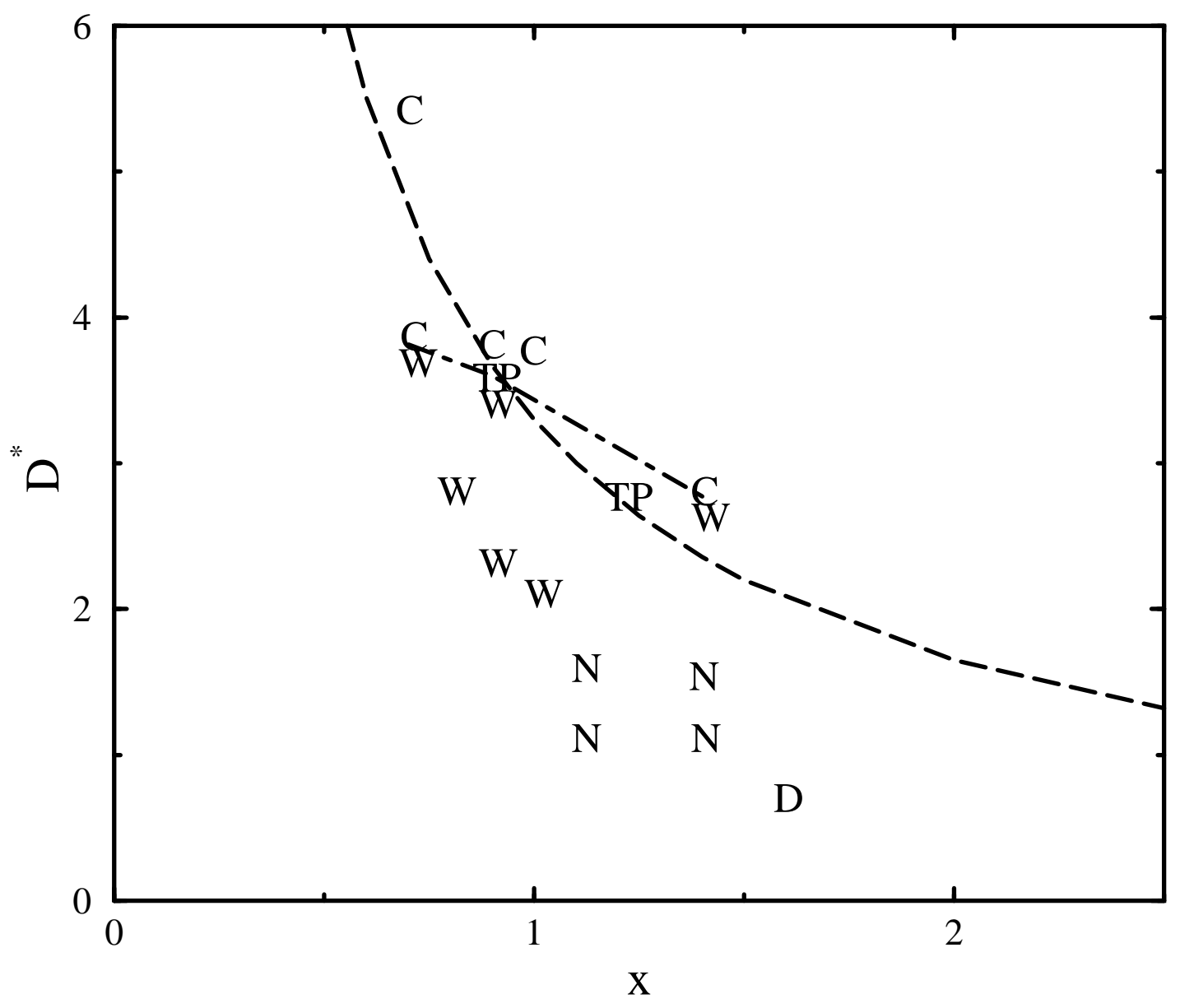

FIG. 8 